\title{
Existence and Stability of Spherically Layered Solutions of the Diblock Copolymer Equation
}

\author{
Xiaofeng Ren ${ }^{* \dagger}$ \\ Department of Mathematics and Statistics \\ Utah State University \\ Logan, UT 84322-3900, USA \\ Juncheng Wei $\$ \S$ \\ Department of Mathematics \\ Chinese University of Hong Kong \\ Shatin, Hong Kong
}

December 1, 2005

\begin{abstract}
The relatively simple Ohta-Kawasaki density functional theory for diblock copolymer melts allows us to construct and analyze exact solutions to the EulerLagrange equation by singular perturbation techniques. First we consider a solution of a single sphere pattern that models a cell in the spherical morphology. We show the existence of the sphere pattern and find a stability threshold, so that if the sphere is larger than the threshold value, the sphere pattern becomes unstable. Next we study a spherical lamellar pattern, which may be regarded as a defective lamellar pattern. We reduce the existence and the stability problems to some finite dimensional problems which are accurately solved with the help of computer. We find two thresholds. Only when the size of the sample is larger than the first threshold value, a spherical lamellar pattern exists. This patten is stable only when the seize of the sample is less than the second threshold value. As the stability of the spherical lamellar pattern changes at the second threshold, a bifurcating branch with a pattern of wriggled spherical interfaces appears. The free energy of the latter pattern is lower than that of
\end{abstract}

*Corresponding author. Phone: 1435 797-0755, Fax 1435 797-1822, E-mail: ren@math.usu.edu † Supported in part by NSF grant DMS-0509725.

‡Phone: 852 2609-7967, Fax 852 2603-5154, E-mail: wei@math.cuhk.edu.hk

$\S$ Supported in part by an Earmarked Grant of RGC of Hong Kong (RGC Proj. 402304). 
the first pattern. A similar bifurcation phenomenon also occurs in the single sphere pattern at its stability threshold.

Keywords: Ohta-Kawasaki diblock copolymer theory, sphere pattern, optimal size, spherical lamellar pattern, existence threshold, stability threshold, bifurcation, wriggled sphere pattern, wriggled spherical lamellar pattern.

AMS subject classifications: 34E05, 82D60.

Short running head: Layered solutions of diblock coplymer equation.

\section{Introduction}

A diblock copolymer melt is a soft material, characterized by fluid-like disorder on the molecular scale and a high degree of order at a longer length scale. A molecule in a diblock copolymer is a linear sub-chain of A-monomers grafted covalently to another sub-chain of B-monomers. Because of the repulsion between the unlike monomers, the different type sub-chains tend to segregate, but as they are chemically bonded in chain molecules, segregation of sub-chains cannot lead to a macroscopic phase separation. Only a local micro-phase separation occurs: micro-domains rich in A monomers and micro-domains rich in $\mathrm{B}$ monomers emerge as a result. These micro-domains form morphology patterns/phases.

There are two types of phase separations in a diblock copolymer system: weak segregation and strong segregation. The weak segregation occurs when the temperature is relatively high. The micro-domains are small and there are no clear interfaces separating them. When the temperature is lower, strong segregation is observed. The micro-domains become larger and they are separated by narrow interfaces.

The self-consistent mean field theory $[11,13,14,15,17,18]$ is the most successful theory in modeling and capturing aspects of the phase separation. It consists of five equations for five field variables: two density fields of A- and B-monomers, two mean fields on A- and B-monomers simulating the interaction between the molecular chains, and a Lagrange multiplier field. Two of the five equations are nonlocal, while the remaining three are algebraic [22]. The theory is derived from a microscopic description of interacting polymer chains. Based on a variational principle, the Gibbs canonical distribution is approximated by the distribution generated by the mean fields [7]. This theory is quite complex to which only numerical studies have been done. One of them is the spectral method of Matsen and Schick [22] that yields predictions with striking resemblances to experiments.

A limitation of such techniques or other test field based methods is that they proceed by assuming a periodic structure, computing its free energy and then comparing that free energy to the free energy of other candidate structures [3]. The patterns found by such methods in general do not exactly solve the self-consistent 
equations. However finding analytic solutions to these equations is very difficult due to the complexity of the two nonlocal equations.

The density functional theory of Ohta and Kawasaki [28] is a much simpler model. The free energy of a diblock copolymer melt is an elegant functional of the A-monomer density field only. Unlike an earlier density functional theory of Leibler [20] that only deals with the weak segregation region, the Ohta-Kawasaki theory deals with both the weak- and strong-segregation phenomena. The Euler-Lagrange equation of the OhtaKawasaki free energy is an integro-differential equation (See (2.9)), which can also be viewed as a system of two elliptic partial differential equations (See (2.11-2.12)).

A close examination of the derivation of the density functional theory shows that it is a simplified version of the self-consistent mean field theory. We refer the reader to [7] for a detailed study of the simplification procedure. Here we briefly summarize the results of [7]. There are two approximation steps. First we consider the relationship between the A-monomer density field $u_{a}$, the B-monomer density field $u_{b}$, and the mean fields $U_{A}, U_{B}$ that act respectively on A- and B- monomers. In the self-consistent mean field theory one can express $u_{a}$ and $u_{b}$ in terms of $U_{A}$ and $U_{B}$ with the help of Feynman integrals, i.e. by solving some parabolic partial differential equations. In the density functional theory we simplify this relationship via linearization. This approximation is accurate if the temperature is not too low. Then we reverse the linearized relationship between $u_{a}, u_{b}$ and $U_{A}, U_{B}$ to express $U_{A}, U_{B}$ in terms of $u_{a}$, $u_{b}$. An analysis in the Fourier space shows that this reversed relationship is described by a pseudo-differential, nonlocal operator. In the second approximation step we keep the long wave and the short wave parts of this operator and discard the intermediate wave effects. This way we end up with a sum of the Laplace operator $-\Delta$ and the inverse Laplace operator $(-\Delta)^{-1}$. When we finally express the free energy as a functional of $u_{a}$ and $u_{b}$ in the density functional theory, the Laplace operator gives rise to the local part of the functional and the inverse Laplace operator leads to the nonlocal part of the functional (See (2.2)).

Despite the shortcomings associated with these approximations, the density functional theory at least qualitatively captures the properties of diblock copolymers $[21,10]$. Ohta and Kawasaki used their theory to study the common lamellar, cylindrical and spherical phases [28]. More recently Teramoto and Nishiura found the less common double gyroid morphology by numerical simulating the theory [43]. Although Ohta and Kawasaki only applied their theory to test fields and did not construct exact solutions of the Euler-Lagrange equation, we will show that the simplicity of the theory actually makes it possible to study exact solutions analytically.

The weak segregation regime may be studied by the bifurcation theory rather easily. One starts with a uniform state and linearizes the Euler-Lagrange equation at the uniform state. For some parameter values the principal eigenvalue of the linearized problem is zero. Then a non-uniform state bifurcates from the uniform state. If one can show that this non-uniform state is stable, then it gives the profile of a weakly segregated pattern. 


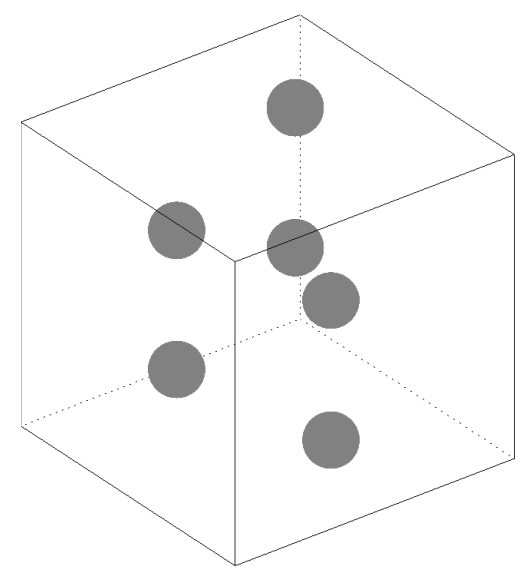

Figure 1: The spherical phase. The B-monomers form dark spheres and the Amonomers occupy the background. Not reflected in this figure is the body centered cubic pattern in which the spheres pack.

In this paper we use the density functional theory to study the more complex strong segregation phenomenon. Strongly segregated patterns are too different from the uniform state to be treated as their bifurcating branches. The appropriate mathematical tool is the singular perturbation theory in calculus of variations and differential equations. We find exact solutions, or at least leading order terms of exact solutions, to the Euler-Lagrange equation of the free energy functional [26, 30, 32, $31,35,39,40,9,6,16]$. Often these solutions may be carefully analyzed and their stability in space can be determined [33, 38].

The first strongly segregated pattern we study is the single sphere pattern. This pattern arises from the spherical phase of a diblock copolymer. When the monomer fraction is skewed in favor of A-monomers, the B-monomers form spherical microdomains, Figure 2. We find a pattern with one sphere of B-monomers as a solution to the Euler-Lagrange equation. There is an optimal size for the sphere. The sphere of the optimal size has lower free energy than those of other spheres. Then we linearize the Euler-Lagrange equation at the sphere solution and study the spectrum of the linearized operator. We will show that there is an upper bound for the size of the sphere. Beyond the upper bound, the sphere can not be stable.

The second pattern is the spherical lamellar pattern. This pattern may be viewed as a defective lamellar pattern. Other defective patterns are considered in [44] where a model of a forth order differential equation is used. Given the number of interfaces we look for a solution that consists of spherical layers of micro-domains separated by narrow interfaces. In this case the existence and stability problems are reduced by singular perturbation techniques to some finite dimensional problems. The reduced problems are easily solved with the help of computer. Note that here we apply numerical methods to the reduced finite dimensional problems only. This way we obtain 
far more accurate and reliable results compared to results found from direct numerical simulations of infinite dimensional problems. There is an existence threshold. Only when the sample is greater than this threshold a spherical lamellar pattern exists. There is also a stability threshold which is greater than the existence threshold. When the sample is larger than the stability threshold, the spherical lamellar pattern becomes unstable.

We emphasize that all the results presented in this paper are mathematically rigorous. The informal style adopted here when we describe perturbation expansions can be changed to a strict mathematical framework, part of which is known as the $\Gamma$-convergence theory $[8,24,23,19]$.

\section{Free energy}

We review the density functional theory of Ohta and Kawasaki [28] in this section. We consider a diblock copolymer melt that occupies a region $D$ in space. The system has the following parameters.

1. The polymerization index $N$ that is the number of all the monomers in a chain molecule.

2. The A-monomer number $N_{A}$ and the B-monomer number $N_{B}$ in a chain. Note that $N_{A}+N_{B}=N$.

3. The number of chain molecules in the melt $n$. The average total monomer number density is $\rho_{0}=\frac{n N}{V}$.

4. The Kuhn statistical length $l$ measuring the average distance between two adjacent monomers in a chain molecule.

5. The inverse absolute temperature $\beta$.

6. The dimensionless Flory-Huggins parameter $\chi$ that measures the repulsion between unlike monomers; it is defined by

$$
\chi=\beta\left(V_{A B}-\frac{V_{A A}+V_{B B}}{2}\right)
$$

where $V_{A B}$ (and $V_{A A}, V_{B B}$ respectively) is the energy cost to bring an Amonomer (A-monomer and B-monomer respectively) and a $\mathrm{B}$ monomer (Amonomer and B-monomer respectively) close to each other. This number is positive because the repulsion force between unlike monomers is stronger than those between like ones. Note that $\chi$ is inversely proportional to temperature.

7. The volume $V$ of the sample. The domain $D$ is non-dimensionalized so that the size of $D$, denoted by $|D|$ is a convenient value. In this paper $D$ is a ball so we take the radius of $D$ to be 1 and the size of $D$ to be $|D|=4 \pi / 3$. 
The main field in the Ohta-Kawasaki theory is the relative A-monomer number density field $u(x)$. The melt is assumed to be incompressible, so when $u(x)=1$ (or $u(x)=0$, respectively), the point $x$ in $D$ is occupied by A-monomers only (or B-monomers only, respectively); if $0<u(x)<1$, a mixture of A- and B-monomers occupies $x$. The free energy $F$ of the system is a functional of $u(x)$. In a dimensionless form we write

$$
\frac{\beta F}{\chi \rho_{0} V}=\int_{D}\left[\frac{\epsilon^{2}}{2}|\nabla u|^{2}+W(u)+\frac{\epsilon \gamma}{2}\left|(-\Delta)^{-1 / 2}(u-a)\right|^{2}\right] d x .
$$

On the right side of (2.2) we have introduced three dimensionless parameters:

$$
\begin{aligned}
\epsilon^{2} & =\frac{|D|^{2 / 3} l^{2}}{12 a(1-a) \chi V^{2 / 3}} \\
\gamma & =\frac{18 \sqrt{3} V}{|D| a^{3 / 2}(1-a)^{3 / 2} \chi^{1 / 2} N^{2} l^{3}} \\
a & =\frac{N_{A}}{N} .
\end{aligned}
$$

Note that the parameter $a=N_{A} / N$ is the average A-monomer density. The field $u$ must satisfy the constraint

$$
\bar{u}=a
$$

where $\bar{u}=\frac{1}{|D|} \int_{D} u(x) d x$ is the average of $u$.

The exact form of $W$ is not given in [28]. In [7] an approximation

$$
W(u)= \begin{cases}u-u^{2} & \text { if } u \in[0,1] \\ \infty & \text { otherwise }\end{cases}
$$

is found. A more accurate $W$ should be a smooth double well function of equal depth. It must have a global minimum value 0 achieved at 0 and 1 . It must have the symmetry $W(u)=W(1-u)$. 0 and 1 are non-degenerate: $W^{\prime \prime}(0)=W^{\prime \prime}(1)>0$.

Central in (2.2) is the third term in the integrand. It is nonlocal and models the long range interaction between monomers due to the connectivity of the molecular chains. The operator $(-\Delta)^{-1 / 2}$ is the square root of the inverse of $-\Delta$ with the natural boundary condition. Alternatively in (2.2) one may write

$$
\int_{D}\left|(-\Delta)^{-1 / 2}(u-a)\right|^{2} d x=\int_{D} \int_{D}(u(x)-a) G(x, y)(u(y)-a) d x d y
$$

where $G$ is the Green function of $-\Delta$ with the natural boundary condition.

The second term in (2.2) can be regarded as the internal energy field of the system, and the first and the third terms give the entropy of the system. As mentioned in the introduction we have only taken the long wave and short wave effects, modeled by the $\nabla$ and $(-\Delta)^{-1 / 2}$ operators, into consideration in this model. 
When we minimize (2.2), the first term in the integrand of (2.2) penalizes any space non-uniformity. The second term favors $u$ being either close to 0 or close to 1 everywhere. The best profile for the third term is to have $u$ close to $a$ everywhere. However this is not a good profile for the second term. The second best profile for the third term is for $u$ to have many oscillations. Local minimizers of the free energy result from these three competing preferences.

The Euler-Lagrange equation of (2.2) is a nonlinear integro-differential equation:

$$
-\epsilon^{2} \Delta u+f(u)+\epsilon \gamma(-\Delta)^{-1}(u-a)=\eta \text { in } D
$$

subject to the natural boundary condition

$$
\partial_{\nu} u=0 \text { on } \partial D
$$

Here $f=W^{\prime}$. The constant $\eta$ on the right side of (2.9) is a Lagrange multiplier coming from the constraint (2.6). The equation (2.9) may also be written as a system of elliptic partial differential equations:

$$
\begin{aligned}
-\epsilon^{2} \Delta u+f(u)+\epsilon \gamma v & =\eta \\
-\Delta v & =u-a
\end{aligned}
$$

subject to the conditions

$$
\partial_{\nu} u=\partial_{\nu} v=0 \text { on } \partial D, \bar{u}-a=\bar{v}=0 .
$$

Note that (2.9) always has the uniform solution $u(x)=a$. When $\epsilon$ is large, corresponding to high temperature, this solution is stable and it models the disordered phase. One may decrease $\epsilon$ to a value so that the principal eigenvalue of the linearized problem at $u(x)=a$ becomes 0 . Then one finds a non-uniform solution bifurcating out of the uniform solution. This bifurcation solution explains the weak segregation phenomenon and the corresponding $\epsilon$ identifies the parameter range for weak segregation.

However in the strong segregation regime $\epsilon$ is much smaller. In this case the free energy (2.2) is most easily analyzed in the parameter range

$$
\begin{aligned}
\epsilon & \ll 1 \\
\gamma & \sim 1 .
\end{aligned}
$$

Here the uniform solution $u(x)=a$ has much higher free energy than those of many other states and is hence thermodynamically un-favored. Under (2.14-2.15), we are in the strong segregation regime and have taken the volume of the sample to be of order

$$
V \sim a^{3 / 2}(1-a)^{3 / 2} \chi^{1 / 2} N^{2} l^{3} .
$$

We will see that in the parameter range (2.14-2.15) the number of micro-domains is of order 1 . Therefore the right side of (2.16) also predicts the size of a micro-domain. 


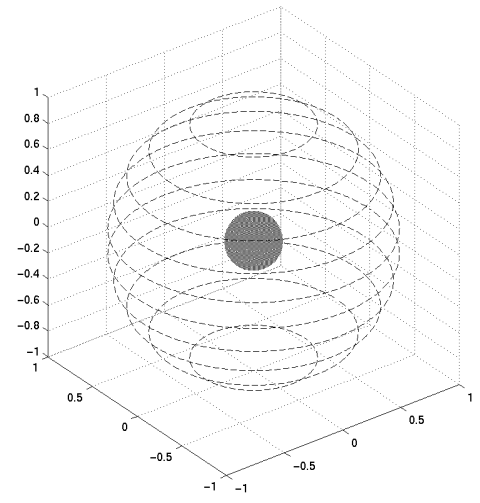

Figure 2: A single sphere solution with the natural boundary condition in the unit ball.

Particularly we find the domain size $V^{1 / 3}$ to be proportional to $N^{2 / 3}$, which is the celebrated $N^{2 / 3}$ law [28].

Having a small $\epsilon$ makes (2.9) a singular perturbation problem. Although in mathematics the singular perturbation theory is much harder and less mature than the regular perturbation theory, a great deal of quantitative properties of solutions to (2.9) can be obtained, using the existing techniques in the theory. Many problems can be solved exactly in the leading order, and many other problems can be reduced to much simpler finite dimensional problems that are solved with the help of computer.

\section{Sphere pattern}

When the monomer fraction $a$ is close to 1 , the diblock polymer typically exists in the spherical phase. B-rich micro-domains form spheres and pack in a body centered cubic pattern (BCC). Here we study a single sphere, Figure 2, based on the model (2.2). Mathematically this must be done before we can analyze the BCC pattern. In a future publication we will "connect" several single sphere patterns to construct a $\mathrm{BBC}$ pattern solution in a general domain.

\subsection{Existence}

When one takes the domain $D$ to be a unit ball, a radially symmetric solution $u(r)$ of (2.9) is found where $u$ now is a function of $r=|x|$, Figure 2. A narrow interface, whose thickness is of order $\epsilon$, exists at some $r_{1}$ where $u\left(r_{1}\right)=1 / 2$. The leading order of $r_{1}$ is determined by (2.6):

$$
r_{1}=(1-a)^{1 / 3}+O(\epsilon)
$$


Inside the interface $u(r)$ is close to 0 and outside $u(r)$ is close to 1 . The profile of $u$ near $r_{1}$ is described by the inner expansion

$$
u(r)=H\left(\frac{r-r_{1}}{\epsilon}\right)+\epsilon P\left(\frac{r-r_{1}}{\epsilon}\right)+O\left(\epsilon^{2}\right) .
$$

The leading order term $H$ is the solution of

$$
-H^{\prime \prime}+f(H)=0
$$

with the conditions $H(-\infty)=0, H(\infty)=1$, and $H(0)=1 / 2$. The next term $P$ is the solution of

$$
-P^{\prime \prime}+f^{\prime}(H) P-\frac{2 H^{\prime}}{r_{1}}+\frac{2 \tau}{r_{1}}=0, P(0)=0 .
$$

The definition of $P$ involves $r_{1}$. Because $1 / r_{1}$ is the mean curvature of the interface, the curvature affects the inner expansion of $u$ in the $\epsilon$ order but not in the leading order, for $H$ is independent of $r_{1}$.

In (3.20) we have a constant $\tau$, which is the interface tension. For a general $W$,

$$
\tau=\int_{0}^{1} \sqrt{2 W(s)} d x
$$

and for (2.7) we have

$$
\tau=\frac{\sqrt{2} \pi}{8}
$$

The interface tension may also be calculated in the self-consistent mean field theory [12]. The value obtained there differs slightly from (3.22) of the density functional theory.

The free energy of this solution may be viewed as a sum of two parts. The first part comes from the two local terms of (2.2) and is equal to

$$
4 \pi r_{1}^{2} \tau \epsilon+O\left(\epsilon^{2}\right)
$$

Note that the first term on the right side is the area of the interface times $\tau$ times $\epsilon$.

The second part of the free energy comes from the nonlocal term of (2.2) and is equal to

$$
\frac{2 \pi r_{1}^{5}\left(r_{1}^{3}-3 r_{1}+2\right) \gamma \epsilon}{15}+O\left(\epsilon^{2}\right)
$$

Note that the free energy of the disordered phase modeled by the uniform solution $u(x)=a$ is $W(a)|D|$, a quantity of order 1 , which is much larger than the free energy of the sphere pattern solution which is of order $\epsilon$. Hence under the condition (2.142.15) the system is in an ordered phase. 


\begin{tabular}{|r|r|r|r|r|r|r|r|r|r|r|}
\hline$a$ & .5 & .525 & .55 & .575 & .6 & .625 & .65 & .675 & .7 & .725 \\
\hline$\gamma_{o}$ & 140 & 130 & 122 & 115 & 109 & 104 & 100 & 97 & 95 & 93 \\
\hline \hline$a$ & .75 & .775 & .8 & .825 & .85 & .875 & .9 & .925 & .95 & .975 \\
\hline$\gamma_{o}$ & 92 & 92 & 93 & 95 & 99 & 106 & 117 & 137 & 176 & 290 \\
\hline
\end{tabular}

Table 1: The values of $\gamma_{o}$ for various $a$.

\subsection{Optimal size}

There is a sphere pattern solution of (2.9) for any $\gamma$ as long as $\epsilon$ is sufficiently small. This means that there is a solution for a wide range of $V$ of the sample (the volume of the B-monomer sphere in the middle is then $(1-a) V)$. It is natural to ask for which value of $V$ the sphere pattern is most energetically favored. Intuitively we know that $V$ can not be too large or too small. By (2.3-2.4) we write $\epsilon=\tilde{\epsilon} V^{-1 / 3}$ and $\gamma=\tilde{\gamma} V$ so that $\tilde{\epsilon}$ and $\tilde{\gamma}$ no longer depend on $V$. Then by (3.23-3.24) we find that the leading term of the re-scaled free energy of a sphere pattern is

$$
4 \pi r_{1}^{2} \tau \tilde{\epsilon} V^{-1 / 3}+\frac{2 \pi r_{1}^{5}\left(r_{1}^{3}-3 r_{1}+2\right) \tilde{\gamma}}{15} \tilde{\epsilon} V^{2 / 3}
$$

With respect to $V,(3.25)$ is minimized at

$$
V=V_{o}=\frac{15 \tau}{r_{1}^{3}\left(r_{1}^{3}-3 r_{1}+2\right) \tilde{\gamma}}
$$

The optimal size of the sample is now given by (3.26). It is more convenient to express this in terms of the dimensionless $\gamma$. The optimal $\gamma$ is denoted by $\gamma_{o}$ which is just

$$
\gamma_{o}=\tilde{\gamma} V_{o}=\frac{15 \tau}{r_{1}^{3}\left(r_{1}^{3}-3 r_{1}+2\right)} .
$$

Table 1 reports the values of $\gamma_{o}$ for various $a$.

\subsection{Stability}

We return to a sphere pattern solution with a general $\gamma$ which is not necessarily equal to $\gamma_{o}$. Although a sphere solution of (2.9) is found for every $\gamma$, we will see that it is stable only if $\gamma$ is not too large. A stable solution of (2.9) is a free energy local minimizer, which corresponds to a meta-stable state of the physical system. An unstable solution can not be observed in experiments.

The stability analysis requires that we solve the eigenvalue problem

$$
-\epsilon^{2} \Delta \varphi+f^{\prime}(u) \varphi-\overline{f^{\prime}(u) \varphi}+\epsilon \gamma(-\Delta)^{-1} \varphi=\lambda \varphi .
$$


The left side of (3.28) comes from linearizing the Euler-Lagrange equation (2.9) at a sphere pattern solution $u$. The eigenvalues $\lambda$ are classified by the mode $l=0,1,2, \ldots$. The eigenvalues whose modes are $l$ are denoted by $\lambda_{l}$. Their corresponding eigenfunctions take the form

$$
\varphi(x)=\phi_{l}(r) Y_{l m}(\theta, \omega)
$$

where $m=0, \pm 1, \ldots, \pm l$, and the $Y_{l m}$ 's are the spherical harmonics. An eigenvalue either approaches 0 , a critical eigenvalue, or stays positively away from 0 when $\epsilon \rightarrow 0$. Hence it suffices to consider the critical eigenvalues.

For the $l=0$ mode there is one critical eigenvalue of order $\epsilon$. It is of multiplicity 1 and has the form

$$
\lambda_{0}=\frac{3 f^{\prime}(0) r_{1}^{2} \epsilon}{\tau}+O\left(\epsilon^{2}\right) .
$$

This eigenvalue is positive, and $l=0$ is a stable mode. The eigenfunction associated with this eigenvalue is radially symmetric. We denote it by $\phi_{0}(r)$. It has the expansion

$$
\phi_{0}(r)=H^{\prime}\left(\frac{r-r_{1}}{\epsilon}\right)+\epsilon P^{\prime}\left(\frac{r-r_{1}}{\epsilon}\right)-\overline{\left[H^{\prime}\left(\frac{r-r_{1}}{\epsilon}\right)+\epsilon P^{\prime}\left(\frac{r-r_{1}}{\epsilon}\right)\right]}+O\left(\epsilon^{2}\right) .
$$

Here $H^{\prime}$ and $P^{\prime}$ are the derivatives of $H$ and $P$, defined in (3.19-3.18), respectively.

For $l=1$ there is one critical eigenvalue of order $\epsilon^{2}$. It has multiplicity 3 and is of the form

$$
\lambda_{1}=\frac{\gamma r_{1}^{4} \epsilon^{2}}{\tau}+O\left(\epsilon^{3}\right) .
$$

This mode is again stable. The eigenfunctions associated with this eigenvalue are $(x / r) \phi_{1}(r),(y / r) \phi_{1}(r)$, and $(z / r) \phi_{1}(r)$ where $\phi_{1}$ has the expansion

$$
\phi_{1}(r)=H^{\prime}\left(\frac{r-r_{1}}{\epsilon}\right)+\epsilon P^{\prime}\left(\frac{r-r_{1}}{\epsilon}\right)+O\left(\epsilon^{2}\right) .
$$

For each $l$ greater than 1 , there is one critical eigenvalue of order $\epsilon^{2}$. This eigenvalue has multiplicity $2 l+1$ and has the form

$$
\lambda_{l}=\left[\frac{l(l+1)-2}{r_{1}^{2}}+\frac{\gamma}{\tau}\left(\frac{r_{1}^{4}-r_{1}}{3}+\frac{(l+1) r_{1}^{2 l+2}}{l(2 l+1)}+\frac{r_{1}}{2 l+1}\right)\right] \epsilon^{2}+O\left(\epsilon^{3}\right) .
$$

The quantity in (3.34) may not always be positive. One finds a threshold $\gamma_{s}$ so that when $\gamma<\gamma_{s}$ all the eigenvalues in (3.34) are positive, but when $\gamma>\gamma_{s}$ at least for one $l$ the eigenvalue $\lambda_{l}$ in (3.34) is negative. Therefore the sphere solution $u$ is stable if $\gamma<\gamma_{s}$ and unstable if $\gamma>\gamma_{s}$. The eigenfunctions associated with $\lambda_{l}$ are $\phi_{l}(r) Y_{l m}$ with $m=0, \pm 1, \ldots, \pm l . \phi_{l}$ has the same expansion as in (3.33).

The leading order of $\gamma_{s}$ is determined from (3.34) following these steps:

1. For each $l \geq 2$, set the leading term

$$
\frac{l(l+1)-2}{r_{1}^{2}}+\frac{\gamma}{\tau}\left(\frac{r_{1}^{4}-r_{1}}{3}+\frac{(l+1) r_{1}^{2 l+2}}{l(2 l+1)}+\frac{r_{1}}{2 l+1}\right)
$$




\begin{tabular}{|r|r|r|r|r|r|r|r|r|r|r|}
\hline$a$ & .5 & .525 & .55 & .575 & .6 & .625 & .65 & .675 & .7 & .725 \\
\hline$\gamma_{s}$ & 463 & 425 & 372 & 336 & 312 & 296 & 276 & 250 & 234 & 225 \\
\hline$l$ & 5 & 5 & 4 & 4 & 4 & 4 & 3 & 3 & 3 & 3 \\
\hline
\end{tabular}

\begin{tabular}{|r|r|r|r|r|r|r|r|r|r|r|}
\hline$a$ & .75 & .775 & .8 & .825 & .85 & .875 & .9 & .925 & .95 & .975 \\
\hline$\gamma_{s}$ & 222 & 225 & 232 & 216 & 209 & 215 & 237 & 283 & 387 & 714 \\
\hline$l$ & 3 & 3 & 3 & 2 & 2 & 2 & 2 & 2 & 2 & 2 \\
\hline
\end{tabular}

Table 2: The (leading order) values of $\gamma_{s}$ for various $a$ and the corresponding mode $l$.

in (3.34) to be 0 , and solve for $\gamma$. Denote the solution for $\gamma$ by $\hat{\gamma}_{l}$. If this $\hat{\gamma}_{l}$ is less than or equal to 0 , the mode $l$ does not yield a zero eigenvalue. Discard such $\hat{\gamma}_{l}$.

2. Minimize the remaining $\hat{\gamma}_{l}$ 's from the last step with respect to $l$. The minimum is achieved at a $\hat{\gamma}_{l}$ which is the leading order of $\gamma_{s}$.

Table 2 reports the leading order of $\gamma_{s}$ for various $a$. At $\gamma=\gamma_{s}$ the smallest eigenvalue is 0 . The mode $l$ of this eigenvalue is also given in Table 2 .

We compare the stability threshold $\gamma_{s}$ to the optimal size $\gamma_{o}$ in Table 1. All the $\gamma_{o}$ 's are significantly less than the corresponding $\gamma_{s}$ 's. Therefore, not surprisingly, the sphere with optimal size is stable.

\section{Spherical lamellar pattern}

When $a$ is close to $1 / 2$, the diblock copolymer exists in the lamellar phase. The perfect lamellar pattern consists micro-domains separated by parallel flat planes, Figure 3. However one often observes the lamellar pattern with topological defects such as dislocations, disclinations, grain boundaries, and tilt boundaries [44]. In this section we consider the spherical lamellar pattern, Figure 4, which we view as a defective lamellar pattern.

Because it involves many interfaces, the study in this section is more complex. Nevertheless we will show that by singular perturbation argument, solving the EulerLagrange equation (2.9) and analyzing the stability of the solution are reduced to studying some finite dimensional problems.

\subsection{Existence}

Unlike the existence problem for the sphere pattern where no condition on $\gamma$ is needed, the existence of a spherical lamellar pattern as a solution of (2.9) requires that $\gamma$ is not too small. We now have an existence threshold $\gamma_{K, e}$. Given the number of interfaces 


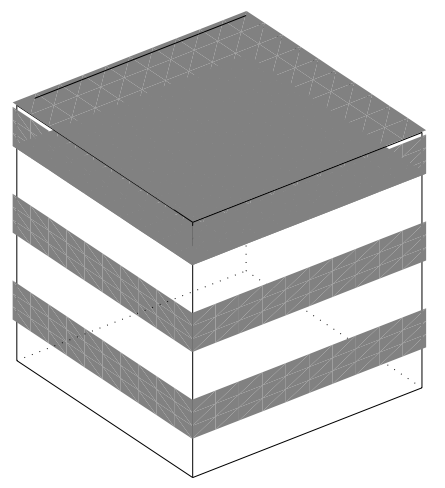

Figure 3: A perfect lamellar pattern.

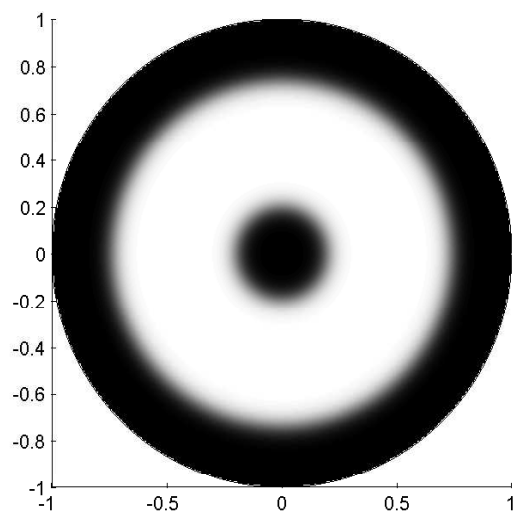

Figure 4: A cross section of a spherical lamellar pattern with two interfaces. 
$K \geq 2$ a $K$-interface spherical lamellar pattern solution of (2.9) exists if $\gamma>\gamma_{K, e}$. If $\gamma<\gamma_{K, e}$ there is no $K$-interface spherical lamellar solution.

When $\gamma>\gamma_{K, e}$, we define the interfaces $r_{j}, j=1,2, \ldots, K$, to be the radii where $u\left(r_{j}\right)=1 / 2$. They have the expansion

$$
r_{j}=r_{j}^{0}+O(\epsilon)
$$

The leading order $r_{j}^{0}$ 's are determined by solving a system of $K+1$ nonlinear equations

$$
\begin{aligned}
\frac{\tau}{r_{j}^{0}}+\frac{(-1)^{j} \gamma}{2} \mathcal{V}\left(r_{j}^{0} ; r_{1}^{0}, r_{2}^{0}, \ldots, r_{K}^{0}\right) & =(-1)^{j} \eta^{0}, \quad j=1,2, \ldots, K \\
\sum_{j=1}^{K}(-1)^{j}\left(r_{j}^{0}\right)^{3}+\frac{1-(-1)^{K}}{2} & =a
\end{aligned}
$$

for $r_{1}^{0}, r_{2}^{0}, \ldots, r_{K}^{0}$, and $\eta^{0}$. Here $\eta^{0}$ is a Lagrange multiplier. The function $\mathcal{V}$ in $(4.37)$ is the solution of

$$
-\mathcal{V}^{\prime \prime}-\frac{2}{r} \mathcal{V}=\mathcal{U}-a, \mathcal{V}^{\prime}(0)=\mathcal{V}^{\prime}(1)=0, \overline{\mathcal{V}}=0
$$

where

$$
\mathcal{U}(r)=0, \text { if } r \in\left(0, r_{1}^{0}\right),=1 \text { if } r \in\left(r_{1}^{0}, r_{2}^{0}\right), \ldots
$$

Denote this solution by $\mathcal{V}\left(r ; r_{1}^{0}, \ldots, r_{K}^{0}\right)$ where we emphasize in its arguments that $\mathcal{V}$ depends on $r_{1}^{0}, \ldots, r_{K}^{0}$. In (4.37) this $\mathcal{V}$ is evaluated at $r=r_{j}^{0}$.

The system (4.37-4.37) is the Euler-Lagrange equations of the minimizer of the function

$$
J\left(q_{1}, q_{2}, \ldots, q_{K}\right)=3 \tau \sum_{k=1}^{K} q_{k}^{2}+\frac{3 \gamma}{2} \int_{0}^{1} \mathcal{V}^{\prime}\left(r ; q_{1}, \ldots, q_{K}\right)^{2} r^{2} d r
$$

subject to the constraint

$$
-q_{1}^{2}+q_{2}^{3}+\ldots+(-1)^{K} q_{K}^{3}+\frac{1-(-1)^{K}}{2}=a .
$$

In the mathematics literature $J$ is known as the $\Gamma$-limit of $(4 \pi \epsilon / 3)^{-1} I$. The $\Gamma$-limit theory thus reduces the study of the infinite dimensional problem $I$ to the study of the finite dimensional problem $J[30,31]$.

Whether $J$ has a minimizer depends on $\gamma$. In general $J$ has a minimizer only if $\gamma$ is large. The border line is exactly the leading order of $\gamma_{K, e}$. For $K=2$, Table 3 reports the leading order of $\gamma_{2, e}$ for various $a$.

Let us consider the $K=2$ case in more detail. We introduce $y$ so that

$$
y=q_{1}^{3}, y+a=q_{2}^{3} .
$$




\begin{tabular}{|}
\begin{tabular}{|r|r|r|r|r|r|r|r|r|r|r|}
\hline$a$ & .5 & .525 & .55 & .575 & .6 & .625 & .65 & .675 & .7 & .725 \\
\hline$\gamma_{2, e}$ & 171 & 175 & 180 & 186 & 194 & 204 & 216 & 230 & 249 & 271 \\
\hline$a$ & .75 & .775 & .8 & .825 & .85 & .875 & .9 & .925 & .95 & .975 \\
\hline$\gamma_{2, e}$ & 300 & 337 & 386 & 453 & 549 & 694 & 932 & 1379 & 2432 & 6590 \\
\hline
\end{tabular}
\end{tabular}

Table 3: The leading order values of $\gamma_{2, e}$ for various $a$.

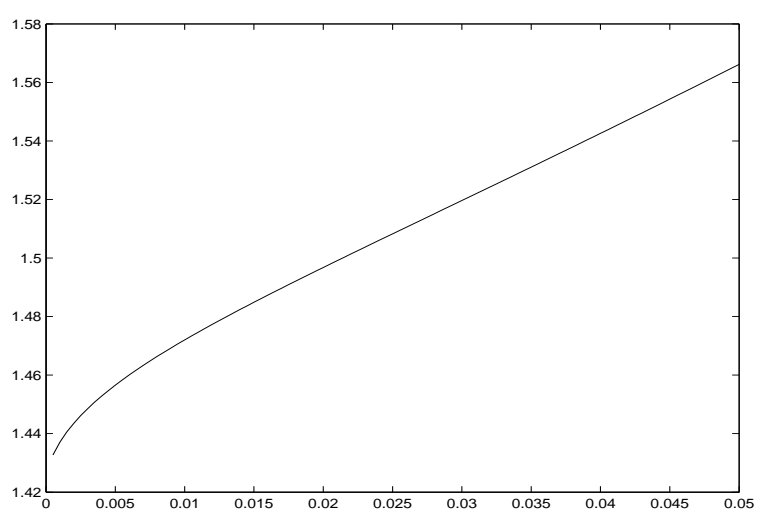

Figure 5: $J$ as a function of $y$ is increasing when $a=1 / 2$ and $\gamma=100$.

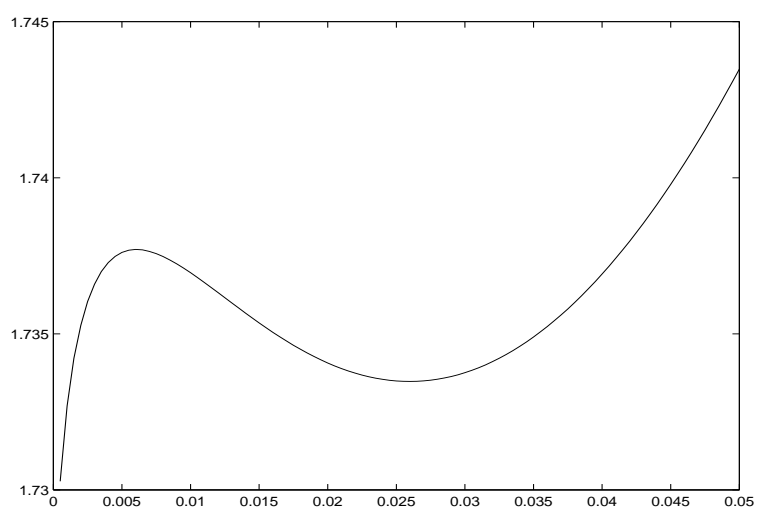

Figure 6: $J$ as a function of $y$ has a local minimum when $a=1 / 2$ and $\gamma=180$. 


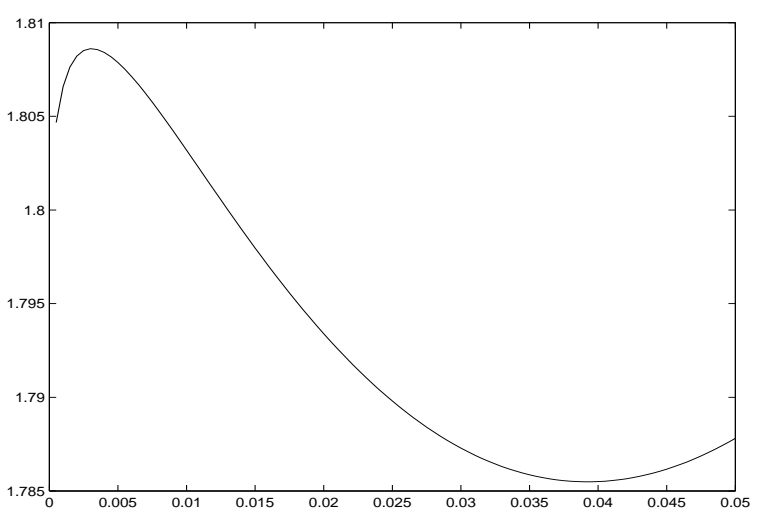

Figure 7: $J$ as a function of $y$ has a global minimum when $a=1 / 2$ and $\gamma=200$.

$J$ can be viewed as a function of $y$ only without the constraint (4.41). Take $a=1 / 2$. For $\gamma=100, J$ is monotonically increasing in $y$, Figure 5. Since $\gamma<\gamma_{2, e} \approx 171$ from Table 3, there is no 2-interface spherical lamellar solution of (2.9).

When $\gamma=180>\gamma_{2, e}, J$ is no longer monotone, Figure 6 . In this case $J$ has a local minimum, and (2.9) has a 2-interface spherical lamellar solution. If we further increase $\gamma$ to 200, the local minimum of $J$ becomes a global minimum, Figure 7 . The spherical lamellar solution continues to exist.

We now return to the general solution with $K$ interfaces. Near each interface $r_{j}$ the solution $u$ again has a profile

$$
u(r)=H\left(\frac{r-r_{j}}{\epsilon}\right)+\epsilon P_{j}\left(\frac{r-r_{j}}{\epsilon}\right)+O\left(\epsilon^{2}\right)
$$

when $j$ is odd and

$$
u(r)=H\left(-\frac{r-r_{j}}{\epsilon}\right)+\epsilon P_{j}\left(-\frac{r-r_{j}}{\epsilon}\right)+O\left(\epsilon^{2}\right)
$$

when $j$ is even. $H$ is the same function defined in (3.19) and $P_{j}$ is define by (3.18) with $r_{1}$ replaced by $r_{j}$.

The free energy of this solution is

$$
\left[4 \pi \tau \sum_{j=1}^{K} r_{j}^{2}+2 \pi \gamma \int_{0}^{1} \mathcal{V}^{\prime}(r)^{2} r^{2} d r\right] \epsilon+O\left(\epsilon^{2}\right) .
$$

\subsection{Stability}

Similar to the sphere pattern solution, a $K$-interface spherical lamellar solution is stable only if $\gamma$ is not too large. More precisely for any given number of interfaces $K$, there is a stability threshold $\gamma_{K, s}$, which is larger than the existence threshold $\gamma_{K, e}$, such that the $K$-interface spherical lamellar solution is stable if $\gamma_{K, e}<\gamma<\gamma_{K, s}$. The solution becomes unstable if $\gamma>\gamma_{K, s}$. 
To verify these statements and determine $\gamma_{K, s}$ we again turn to the linearized problem (3.28). But this time $u$ is the $K$-interface spherical lamellar solution found in Section 4.1. The eigenvalues are again classified by the mode $l=0,1,2, \ldots$ Denote the eigenvalues whose modes are $l$ by $\lambda_{l}$. For each $l$ the non-critical eigenvalues all stay positively away from 0 , so it suffices to find the critical eigenvalues to determine whether $u$ is stable.

When $l$ is equal to 0 , there exist $K$ critical eigenvalues. All of them are simple. One of them is of order $\epsilon$ and has the expansion

$$
\lambda_{0}=\frac{3 f^{\prime}(0) \sum_{k=1}^{K}\left(r_{k}^{0}\right)^{2}}{\tau} \epsilon+O\left(\epsilon^{2}\right),
$$

which is positive. The associated eigenfunction is

$$
\phi_{0}(r)=\sum_{j=1}^{K}\left\{H^{\prime}\left(\frac{r-r_{j}}{\epsilon}\right)+\epsilon P_{j}^{\prime}\left(\frac{r-r_{j}}{\epsilon}\right)-\overline{\left.\left[H^{\prime}\left(\frac{r-r_{j}}{\epsilon}\right)+\epsilon P_{j}^{\prime}\left(\frac{r-r_{j}}{\epsilon}\right)\right]\right\}}+O\left(\epsilon^{2}\right) .\right.
$$

The remaining $K-1$ eigenvalues of mode $l=0$ are of order $\epsilon^{2}$. Let us expand them as

$$
\lambda_{0}=\mu_{0} \epsilon^{2}+O\left(\epsilon^{3}\right) .
$$

The determination of $\mu_{0}$ is more complex.

First we define a $K$ by $K$ matrix $M$ whose $k j$-entry is

$$
\begin{cases}\left(-\frac{2 \tau}{\left(r_{k}^{0}\right)^{2}}+\gamma(-1)^{k} \mathcal{V}^{\prime}\left(r_{k}^{0}\right)\right)+\gamma G_{0}\left(r_{k}^{0}, r_{k}^{0}\right) & \text { if } \quad k=j \\ \gamma G_{0}\left(r_{k}^{0}, r_{j}^{0}\right) & \text { if } \quad k \neq j\end{cases}
$$

where $G_{0}$ is a Green function:

$$
G_{0}(r, s)=\left\{\begin{array}{lll}
\frac{s^{2} r^{2}}{2}+s-\frac{9 s^{2}}{5}+\frac{s^{4}}{2} & \text { if } \quad r<s \\
\frac{s^{2} r^{2}}{2}+\frac{s^{2}}{r}-\frac{9 s^{2}}{5}+\frac{s^{4}}{2} & \text { if } \quad r \geq s
\end{array} .\right.
$$

Then we set a non-standard inner product

$$
\langle A, B\rangle=\sum_{k=1}^{K} A_{k} B_{k}\left(r_{j}^{0}\right)^{2} .
$$

Let $\mathbf{e}_{1}, \mathbf{e}_{2}, \ldots, \mathbf{e}_{K}$ be an orthonormal basis with respect to the inner product (4.51) and

$$
\mathbf{e}_{1}=\frac{(1,1, \ldots, 1)}{\sqrt{\langle(1,1, \ldots, 1),(1,1, \ldots, 1)\rangle}} .
$$

The $\mu_{0}$ 's are determined from a $K-1$ dimensional eigenvalue problem:

$$
\sum_{m=2}^{K} d_{m} N_{m n}=\mu_{0} \tau d_{n}, n=2,3, \ldots, K
$$


The $K-1$ by $K-1$ matrix $N$ is obtained by projecting the $K$ by $K$ matrix $M$ into the $K-1$ dimensional subspace spanned by $\mathbf{e}_{2}, \mathbf{e}_{3}, \ldots, \mathbf{e}_{K}$ :

$$
N_{m n}=\left\langle M \mathbf{e}_{m}, \mathbf{e}_{n}\right\rangle, \quad m, n=2,3, \ldots, K .
$$

The inner product in (4.54) is the one defined in (4.51).

These critical eigenvalues turn out to be all positive. This follows as a consequence of Section 4.1. That $N$ is positive definite is equivalent to the fact that $r_{j}^{0}$ minimizes $J$, defined in (4.40). The latter condition is fulfilled when $\gamma>\gamma_{K, e}$. Hence $l=0$ is a stable mode. To each one of these $k-1 \lambda_{0}$ 's, the corresponding eigenfunction $\phi_{0}(r)$ is given with the help of the eigenvector $\left(d_{2}, d_{3}, \ldots, d_{K}\right)$ of $(4.53)$ :

$$
\phi_{0}(r)=\sum_{j=1}^{K} c_{j}\left[H^{\prime}\left(\frac{r-r_{j}}{\epsilon}\right)+\epsilon P_{j}^{\prime}\left(\frac{r-r_{j}}{\epsilon}\right)\right]+O\left(\epsilon^{2}\right),
$$

where

$$
\left(c_{1}, c_{2}, \ldots, c_{K}\right)=\sum_{n=2}^{K} d_{n} \mathbf{e}_{n} .
$$

When $l$ is greater than 0 , there are $K$ critical eigenvalue for each $l$. They all have multiplicity $2 l+1$. All of them are of order $\epsilon^{2}$. If we write

$$
\lambda_{l}=\epsilon^{2} \mu_{l}+O\left(\epsilon^{3}\right)
$$

then the $\mu_{l}$ 's are found by solving the $K$-dimensional eigenvalue problem

$$
\left[\frac{(l(l+1)-2) \tau}{\left(r_{k}^{0}\right)^{2}}+(-1)^{k} \gamma \mathcal{V}\left(r_{k}^{0}\right)\right] c_{k}+\gamma \sum_{j=1}^{K} G_{l}\left(r_{k}^{0}, r_{j}^{0}\right) c_{j}=\mu_{l} \tau c_{k}, k=1,2, \ldots, K
$$

where $G_{l}$ is another Green function:

$$
G_{l}(r, s)=\left\{\begin{array}{ll}
\left(\frac{s^{1-l}}{2 l+1}+\frac{(l+1) s^{2+l}}{l(2 l+1)}\right) r^{l} & \text { if } \quad r<s \\
s^{2+l}\left(\frac{r^{-1-l}}{2 l+1}+\frac{(l+1) r^{l}}{l(2 l+1)}\right) & \text { if } \quad r \geq s
\end{array} .\right.
$$

These critical eigenvalues are not always positive. There exists a stability threshold $\gamma_{K, s}$ so that when $\gamma_{K, e}<\gamma<\gamma_{K, s}$, all the critical eigenvalues are positive and hence the $K$-interface solution $u$ is stable, and when $\gamma>\gamma_{K, s}$ at lease one critical eigenvalue is negative and the $K$-interface solution $u$ is unstable. To each $\lambda_{l}$ the corresponding eigenfunctions are $\phi_{l}(r) Y_{l m}, m=0, \pm 1, \ldots, \pm l . \phi_{l}$ is determined with the help of the eigenvectors $c_{k}$ in (4.58):

$$
\phi_{l}(r)=\sum_{j=1}^{K} c_{j}\left[H^{\prime}\left(\frac{r-r_{j}}{\epsilon}\right)+\epsilon P_{j}^{\prime}\left(\frac{r-r_{j}}{\epsilon}\right)\right]+O\left(\epsilon^{2}\right) .
$$

Table 4 reports the stability threshold values for various $a$. Note that the $\gamma_{2, s}$ 's are greater than the corresponding $\gamma_{2, e}$. Hence there is a range $\left(\gamma_{2, e}, \gamma_{2, s}\right)$ for $\gamma$ where the 2-interface spherical lamellar pattern is stable. 


\begin{tabular}{|r|r|r|r|r|r|r|r|r|r|r|}
\hline$a$ & .5 & .525 & .55 & .575 & .6 & .625 & .65 & .675 & .7 & .725 \\
\hline$\gamma_{2, s}$ & 1162 & 1067 & 1002 & 956 & 931 & 919 & 924 & 943 & 951 & 939 \\
\hline$l$ & 4 & 3 & 3 & 3 & 3 & 3 & 3 & 3 & 2 & 2 \\
\hline
\end{tabular}

\begin{tabular}{|r|r|r|r|r|r|r|r|r|r|r|}
\hline$a$ & .75 & .775 & .8 & .825 & .85 & .875 & .9 & .925 & .95 & .975 \\
\hline$\gamma_{2, s}$ & 952 & 999 & 1077 & 1199 & 1393 & 1711 & 2241 & 3254 & 5684 & 15454 \\
\hline$l$ & 2 & 2 & 2 & 2 & 2 & 2 & 2 & 2 & 2 & 2 \\
\hline
\end{tabular}

Table 4: The (leading order) values of $\gamma_{2, s}$ for various $a$ and the corresponding mode $l$.

\section{Discussion}

The single sphere pattern studied in Section 3 only gives a limited picture of the spherical phase of a diblock copolymer, where multiple spheres co-exist. Moreover the spheres are observed to pack in the body centered cubic pattern. An analytic study of such a multi-sphere pattern requires more refined singular perturbation techniques. The main difficulty is that the spheres in such a phase are only approximately round. The following argument illustrates this point.

It is known that even in a general domain, which we call $\Omega,(2.9)$ has a singular limit as $\epsilon \rightarrow 0$ [30]. The leading order outer expansion $u^{0}$ of $u$, a solution of (2.9), has the property that for a.e. $x \in \Omega u^{0}(x)=0$ or $u^{0}(x)=1$ and $\overline{u^{0}}=a$. Let $S$ be the union of the interfaces that separate the regions $u^{0}=0$ (B-rich micro-domains) from the regions $u^{0}=1$ (A-rich micro-domains), and $v^{0}=(-\Delta)^{-1}\left(u^{0}-a\right)$. In the singular limit an interface is a two-dimensional surface, with no thickness. At every $x \in S$

$$
\tau \kappa(x)+\gamma v^{0}(x)=\eta^{0}
$$

where $\kappa(x)$ is the mean curvature of $S$ at $x$ viewed from the $u^{0}=1$ side, and $\eta^{0}$ is a Lagrange multiplier to be determined. The equation (5.61) is a generalization of (4.37). The constraint (4.37) is replaced by $\overline{u^{0}}=a$. If the free boundary problem (5.61) admits an isolated stable solution $u^{0}$, then near $u^{0}$ there exists a local minimizer solution $u$ of (2.9). However (5.61) is still a challenging nonlocal geometric problem. Even though Figure 1 (3) suggest that we look for solutions with multiple spheres, (5.61) implies that for such a solution the curvature of the interface of a sphere is in general not constant (there is the impact of $v^{0}$ ), i.e. the spheres are not exactly round, unless we deal with the one sphere or the spherically lamellar solutions in a ball as in this paper.

Nevertheless if we consider the situation where $a$ is close to 0 (or 1 ), then $v^{0}$ is near constant throughout $\Omega$ and hence $\kappa$ becomes close to a constant and the spheres are approximately round. The spherical phase in Figure 1 is thus heuristically explained. One must realize that in the small $a$ case, i.e. small droplet-high curvature case, the parameter $\epsilon$ should be significantly less than $a$, otherwise we can not have 

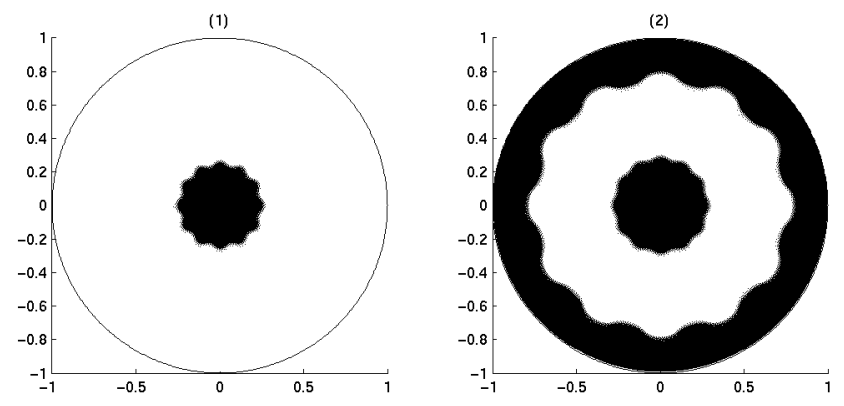

Figure 8: A cross section of a wiggling sphere solution, and a cross section of a wriggled spherical lamellar solution with two interfaces.

morphologies with micro-domains separated by sharp interfaces. It was shown in [40] that the borderline range for $a$ in one-dimension is $a \sim \epsilon^{1 / 2}$. It is not clear at the moment what the borderline values for $a$ are in two and three dimensions.

The stability threshold $\gamma_{s}$ (or $\gamma_{K, s}$ ) is related to a strong segregation bifurcation phenomenon (not to be confused with the bifurcation analysis in the weak segregation regime). When $\gamma$ passes $\gamma_{s}$ (or $\gamma_{K, s}$ ) a second solution bifurcates out of the sphere (or spherical lamellar) solution. The new solution differs from the old one by a quantity which is roughly proportional to the eigenfunction of the 0 principal eigenvalue at $\gamma=\gamma_{s}$ (or $\gamma_{K, s}$ ). Because the eigenfunction has the form (3.29), the new solution has a wiggling interface (or interfaces). The wiggles are determined by the spherical harmonics $Y_{l m}$ in (3.29), Figure 8. Such a wiggling interface solution can be regarded as another defective pattern. If we consider the free energy during the bifurcation process, the bifurcating branch lowers the nonlocal part of the free energy by introducing more oscillation but increases the interface energy. The overall free energy of the bifurcating branch is lower than that of the first branch.

We did not discuss the dynamics of a diblock copolymer system. The purpose of studying the critical eigenvalues of a solution of (2.9) in this paper is to determine whether the solution is a local minimizer of (2.2). However the same critical eigenvalues also determine the local dynamics, near the solution, of the evolution equation

$$
u_{t}=\epsilon^{2} \Delta u-f(u)-\epsilon \gamma(-\Delta)^{-1}(u-a)+\overline{f(u)}, \quad \partial_{\nu} u=0 \text { on } \partial D .
$$

Note that $\int_{D} u(x) d x$ is conserved under (5.62) because $\frac{d}{d t} \int_{D} u(x) d x=0$ after one 
integrates (5.62) over $D$. The eigenfunctions of the critical eigenvalues give the directions along which the dynamics of (5.62) runs slowly (the eigenfunctions of the non-critical eigenvalues are directions of fast dynamics).

The critical eigenvalues in this context admit geometric interpretations. For the sphere pattern the eigenfunction of the critical eigenvalue $\lambda_{0}$ in (3.30) is radially symmetric. So the dynamics in this direction involves only the shape change of $u$ in the radial direction. The eigenfunctions of the critical eigenvalue $\lambda_{1}$ in (3.32) have the forms $(x / r) \phi_{1}(r),(y / r) \phi_{1}(r)$ and $(z / r) \phi_{1}(r)$. They lead to translations of $u$ in $x, y$, or $z$ directions in the dynamics. Finally the eigenfunctions of the critical eigenvalues $\lambda_{l}, l>1$, in (3.34) give rise to oscillations of the interfaces. The same interpretations are also valid for the spherical lamellar pattern. Because all these eigenfunctions concentrate at the interface $r_{j}$ by (3.31), (3.33), (4.47), (4.55) and (4.60), the dynamics along the directions of the critical eigenvalues is seen as the motion of the interfaces.

However (5.62) is only one dynamical law that we can associate to (2.2). A more realistic one is the forth-order partial differential equation [2]

$$
u_{t}=\Delta\left(-\epsilon^{2} \Delta+f(u)\right)-\epsilon \gamma(u-a), \quad \partial_{\nu} u=\partial_{\nu} \Delta u=0 \text { on } \partial D . .
$$

Equation (5.63) generalizes the well-known Cahn-Hilliard equation [5]. Based on a spectral comparison argument [4], one shows that a steady state is stable under (5.62) if and only if it is stable under (5.63) [27]. Hence our results on the stability of the various steady states in this paper remain valid in the dynamics (5.63). An even more complex dynamical law considers a diblock copolymer melt as a fluid. It adds the velocity field and couples (5.63) to the Navier-Stokes equation of the velocity field [2].

We are mainly interested in stable solutions of (2.9). They are local minimizers of (2.2). We do not know whether or not any of the solutions found in this paper is a global minimizer. There also exist unstable spherical lamellar solutions even for $\gamma \in\left(\gamma_{K, e}, \gamma_{K, s}\right)$. In Figures 6 and 7 when $\gamma>\gamma_{2, e}$ in addition to the minimum of $J$ there exists a local maximum of $J$. This maximum point corresponds to a unstable spherical lamellar solution. This unstable solution exists for $\gamma \geq \gamma_{2, s}$ as well. The instability of this solution is caused by the $m=0$ mode.

In the functional (2.2) the key ingredient is the nonlocal term. It describes a long range interaction. Many other important physical systems that exhibit selforganization and pattern formation share the same phenomenon [42]. Examples include charged Langmuir monolayers [1], and smectic liquid crystal films [41]. Many of the singular perturbation techniques presented here may be applied to these problems [29, 34, 37, 36].

The nonlocal interaction in (2.2) is of Coulomb type [25]. Some of the above mentioned problems have different nonlocal interactions. In the charged monolayer problem the nonlocal term is written as

$$
\int_{D} \int_{D}(u(x)-a) G_{c}(x, y)(u(y)-a) d x d y
$$


which assumes the same form as (2.8) does. However the kernel $G_{c}$ is different. If $D$ is a square, i.e. $(0,1)^{2}$, with the periodic boundary condition, then $G_{c}$ is translation invariant so that $G_{c}(x, y)=G_{c}(x-y)$. The Fourier series of $G_{c}$ is

$$
\hat{G}_{c}(\xi)=\frac{1}{|\xi|} \text {. }
$$

Note that for the diblock copolymer problem the corresponding $G$ on a square is

$$
\hat{G}(\xi)=\frac{1}{|\xi|^{2}}
$$

Hence as $|\xi| \rightarrow \infty,(5.66)$ has a faster decay rate than (5.65) does. Many properties, such as the optimal size discussed in Section 3.2, are sensitive to these decay rates. In general with a slower decay rate, one finds smaller micro-domains [34].

In the smectic liquid crystal film problem, the nonlocal interaction comes from a coupling effect with the director field. In this case, because of the unit length constraint on the director field, the nonlocal interaction is no longer quadratic [36].

\section{Conclusion}

We used asymptotic analysis to study the Ohta-Kawasaki density functional theory for diblock copolymers. We constructed a single sphere pattern in a unit ball. Such a pattern is a cell in the spherical morphology. We showed the existence of the sphere pattern as a solution of the Euler-Lagrange equation of the free energy. We identified the optimal size of such a cell with the least free energy. We also found a stability threshold. The sphere is stable if it is less than the threshold value, and unstable if it is greater than the threshold value. The stability threshold value is greater than the optimal size. At the stability threshold, there is another solution, a bifurcating branch. It has an interface of a wriggled sphere. This solution has lower free energy than that of the first solution.

Next we studied a spherical lamellar pattern, which we view as a defective lamellar pattern. Singular perturbation analysis allowed us to reduce the existence and stability problems in infinite dimensions to existence and matrix problems in finite dimensions. We found two thresholds: an existence threshold and a larger, stability threshold. There is a spherical lamellar pattern only when the size of the sample is larger than the existence threshold value. This patten is stable only when the seize of the sample is between the existence threshold and the stability threshold. At the stability threshold, there is a bifurcating branch with a pattern of wriggled spherical interfaces. The bifurcating branch again has lower free energy. 


\section{References}

[1] D. Andelman, F. Broçhard, and J.-F. Joanny. Phase transitions in Langmuir monolayers of polar molecules. J. Chem. Phys., 86(6):3673-3681, 1987.

[2] M. Bahiana and Y. Oono. Cell dynamical system approach to block copolymers. Phys. Rev. A, 41(12):6763-6771, 1990.

[3] F. S. Bates and G. H. Fredrickson. Block copolymers - designer soft materials. Physics Today, 52(2):32-38, 1999.

[4] P.W. Bates and P.C. Fife. Spectral comparison principles for the Cahn-Hilliard and phase-field equations, and times scales for coarsening. Physica D, 43(23):335-348, 1990.

[5] J.W. Cahn and J.E. Hilliard. Free energy of a nonuniform system. I. Interfacial free energy. J. Chem. Phys., 28(2):258-267, 1958.

[6] R. Choksi. Scaling laws in microphase separation of diblock copolymers. J. Nonlinear Sci., 11(3):223-236, 2001.

[7] R. Choksi and X. Ren. On the derivation of a density functional theory for microphase separation of diblock copolymers. J. Statist. Phys., 113(1-2):151176, 2003.

[8] E. De Giorgi. Sulla convergenza di alcune successioni d'integrali del tipo dell'area. Rend. Mat. (6), 8:277-294, 1975.

[9] P. C. Fife and D. Hilhorst. The Nishiura-Ohnishi free boundary problem in the 1D case. SIAM J. Math. Anal., 33(3):589-606, 2001.

[10] I. W. Hamley. The Physics of Block Copolymers. Oxford Science Publications, 1998.

[11] E. Helfand. Theory of inhomogeneous polymers: fundamentals of Gaussian random-walk model. J. Chem. Phys., 62(3):999-1005, 1975.

[12] E. Helfand and Y. Tagami. Theory of the interface between immiscible polymers II. J. Chem. Phys., 56(7):3592-3601, 1972.

[13] E. Helfand and Z. R. Wasserman. Block copolymer theory. 4. narrow interphase approximation. Macromolecules, 9(6):879-888, 1976.

[14] E. Helfand and Z. R. Wasserman. Block copolymer theory. 5. spherical domains. Macromolecules, 11(5):960-966, 1978. 
[15] E. Helfand and Z. R. Wasserman. Block copolymer theory. 6. cylindrical domains. Macromolecules, 13(4):994-998, 1980.

[16] M. Henry. Singular limit of a fourth order problem arising in the micro-phase separation of diblock copolymers. Adv. Differential Equations, 6(9):1049-1114, 2001.

[17] K. M. Hong and J. Noolandi. Theory of inhomogeneous multicomponent polymer systems. Macromolecules, 14(3):727-736, 1981.

[18] K. M. Hong and J. Noolandi. Theory of phase equilibria in systems containing block copolymers. Macromolecules, 16(7):1083-1093, 1983.

[19] R. Kohn and P. Sternberg. Local minimisers and singular perturbations. Proc. Roy. Soc. Edinburgh Sect. A, 111(1-2):69-84, 1989.

[20] L. Leibler. Theory of microphase separation in block copolymers. Macromolecules, 13(6):1602-1617, 1980.

[21] M. W. Matsen and F. S. Bates. Unifying weak- and strong-segregation block copolymer theories. Macromolecules, 29(4):1091-1098, 1996.

[22] M. W. Matsen and M. Schick. Stable and unstable phases of a diblock copolymer melt. Phys. Rev. Letters, 72(16):2660-2663, 1994.

[23] L. Modica. The gradient theory of phase transitions and the minimal interface criterion. Arch. Rat. Mech. Anal., 98(2):123-142, 1987.

[24] L. Modica and S. Mortola. Un esempio di $\Gamma^{-}$-convergenza. Boll. Un. Mat. Ital. $B$ (5), 14(1):285-299, 1977.

[25] C. B. Muratov. Theory of domain patterns in systems with long-range interactions of Coulomb type. Phys. Rev. E, 66:066108, 2002.

[26] Y. Nishiura and I. Ohnishi. Some mathematical aspects of the microphase separation in diblock copolymers. Physica D, 84(1-2):31-39, 1995.

[27] I. Ohnishi and Y. Nishiura. Spectral comparison between the second and fourth order equations of conservative type with non-local terms. Japan J. Indust. Appl. Math., 15(2):253-262, 1998.

[28] T. Ohta and K. Kawasaki. Equilibrium morphology of block copolymer melts. Macromolecules, 19(10):2621-2632, 1986.

[29] X. Ren and L. Truskinovsky. Finite scale microstructures in nonlocal elasticity. In recognition of the sixtieth birthday of Roger L. Fosdick (Blacksburg, VA, 1999). J. Elasticity, 59(1-3):319-355, 2000. 
[30] X. Ren and J. Wei. On the multiplicity of solutions of two nonlocal variational problems. SIAM J. Math. Anal., 31(4):909-924, 2000.

[31] X. Ren and J. Wei. Concentrically layered energy equilibria of the di-block copolymer problem. European J. Appl. Math., 13(5):479-496, 2002.

[32] X. Ren and J. Wei. On energy minimizers of the di-block copolymer problem. Interfaces Free Bound., 5(2):193-238, 2003.

[33] X. Ren and J. Wei. On the spectra of 3-D lamellar solutions of the diblock copolymer problem. SIAM J. Math. Anal., 35(1):1-32, 2003.

[34] X. Ren and J. Wei. Soliton-stripe patterns in charged Langmuir monolayers. J. Nonlinear Sci., 13(6):603-624, 2003.

[35] X. Ren and J. Wei. Triblock copolymer theory: Ordered ABC lamellar phase. J. Nonlinear Sci., 13(2):175-208, 2003.

[36] X. Ren and J. Wei. Chiral symmetry breaking and the soliton-stripe pattern in Langmuir monolayers and smectic films. Nonlinearity, 17(2):617-632, 2004.

[37] X. Ren and J. Wei. The soliton-stripe pattern in the Seul-Andelman membrane. Physica D, 188(3-4):277-291, 2004.

[38] X. Ren and J. Wei. Stability of spot and ring solutions of the diblock copolymer equation. J. Math. Phys., 45(11):4106-4133, 2004.

[39] X. Ren and J. Wei. Wriggled lamellar solutions and their stability in the diblock copolymer problem. SIAM J. Math. Anal., 37(2):455-489, 2005.

[40] X. Ren and J. Wei. Droplet solutions in the diblock copolymer problem with skewed monomer composition. Calc. Var. Partial Differential Equations, in press.

[41] J. V. Selinger, Z.-G. Wang, R. F. Bruinsma, and C. M. Knobler. Chiral symmetry breaking in Langmuir monolayers and smectic films. Phys. Rev. Lett., 70(8):11391142, 1993.

[42] M. Seul and D. Andelman. Domain shapes and patterns: The phenomenology of modulated phases. Science, 267:476-483, 1995.

[43] T. Teramoto and Y. Nishiura. Double gyroid morphology in a gradient system with nonlocal effects. Journal of the Physical Society of Japan, 71(7):1611-1614, 2002 .

[44] Y. Tsori, D. Andelman, and M. Schick. Defects in lamellar diblock copolymers: Chevron- and $\Omega$-shaped boundaries. Phys. Rev. E, 61(3):2848-2858, 2000. 